\title{
TRATAMIENTO CON LA TÉCNICA DE NESBIT EN PACIENTES CON LA ENFERMEDAD DE PEYRONIE
}

\author{
J. RODRÍGUEZ TOLRÁ, E. FRANCO MIRANDA, J.M. PRATS PUIG, O. BUISAN RUEDA, \\ N. SERRALLACH MILA
}

Unidad de Andrología. Servicio de Urología. Ciudad Hospital Universitario de Bellvitge. Hospitalet de Llobregat. Barcelona.

Actas Urol Esp. 27 (10): 803-808, 2003

\section{RESUMEN}

TRATAMIENTO CON LA TÉCNICA DE NESBIT EN PACIENTES CON LA ENFERMEDAD DE PEYRONIE OBJETIVO: Evaluar los resultados obtenidos mediante la técnica de Nesbit en los pacientes con enfermedad de Peyronie.

MATERIAL Y MÉTODOS: Entre enero de 1990 y julio del 2002, hemos aplicado la técnica de Nesbit para la corrección de la incurvación peneana en la enfermedad de Peyronie en 45 pacientes. La edad media era de 57,6 años (Rango 41-73 años ), siendo la incurvación dorso-lateral (42,28\%) la más frecuente.

RESULTADOS: Se consiguió la corrección total de la incurvación en 40 pacientes $(88,88 \%$ ) y un grado de satisfacción elevado por la cirugía en 39 pacientes $(86,66 \%)$.

CONCLUSIONES: La técnica de Nesbit es una intervención quirúrgica sencilla y efectiva para la corrección de la enfermedad de Peyronie.

PALABRAS CLAVE: Técnica de Nesbit. Enfermedad de Peyronie.

\section{ABSTRACT}

NESBIT'S TECHNIQUE TREATMENT IN PATIENTS WITH PEYRONIE DISEASE

OBJECTIVE: Evaluate the results of Nesbit's technique in patients with Peyronie's disease.

METHODS AND MATERIALS: Between 1990 and 2002, 45 patients were treated using the Nesbit's procedure to correct Peyronie's disease curvature.

Mean age was 57.6 (range 41-73). Dorso lateral incurvation was the most comon.

RESULTS: Total correction of the curvature in 40 patients (88\%). High grade of satisfaction in 39 patientes (86.66\%).

CONCLUSION: Nesbit's operation is an easy and effective surgical technique in the peyronie's disease for curvature correction.

KEY WORDS: Nesbit's technique. Peyronie's disease.

$\mathrm{L}^{2}$ a enfermedad de Peyronie es un transtorno del tejido conectivo que afecta a la túnica albuginea de los cuerpos cavernosos. Aparece como una placa que en la fase temprana de su evolución suele ser dolorosa, sobre todo, durante la erección. Provoca además incurvación del miembro, que en grados avanzados impide o dificulta la erección.
Se trata de una enfermedad de etiología desconocida, barajándose entre otras posibilidades: la enfermedad inflamatoria adquirida, la colagenosis difusa con posible base genética, la existencia de factores inmunológicos e incluso el uso de ciertas drogas (fundamentalmente betabloqueantes). 
Su evolución es también paradójica, ya que si bien lo normal es su progresión o estabilización, hay un porcentaje de casos que evolucionan hacia la curación sin secuelas.

$\mathrm{Su}$ principal problema radica en que la incurvación impide o dificulta la penetración, estando reservada para estos casos la cirugía. Ésta puede aplicarse para corregir la incurvación mediante plastias (técnica de Nesbit) o plicaturas, o bien por escisión/incisión de la placa e injerto.

Fue Nesbit ${ }^{1}$ quien en 1965 introdujo el concepto de plastia para el tratamiento de la incurvación congénita del pene (la técnica consiste en la escisión de una o más elipses de la albugínea en el lado convexo, con el fin de acortarlo).

Pryor $^{2}$ en 1979 la utiliza por primera vez en el tratamiento de la enfermedad de Peyronie. Posteriormente han aparecido muchas variantes como la de Kelami ${ }^{3}$ o la de Yachia ${ }^{4}$, que practica una incisión longitudinal y sutura transversal.

\section{MATERIAL Y MÉTODOS}

Entre enero de 1990 y julio del 2002, hemos intervenido 45 pacientes con la enfermedad de Peyronie mediante la técnica de Nesbit. La edad media fue de 57,6 años (Rango 41-73 años). El motivo de la intervención en todos los casos fue la dificultad o imposibilidad para la penetración debido a la incurvación del pene que provoca la enfermedad. La imposibilidad la vimos en 25 casos $(55,55 \%)$ y la dificultad en 20 casos $(44,44 \%)$. El tipo de incurvación viene reflejado en la Tabla I.

Tanto para valorar el grado de la incurvación como el tipo de ésta empleamos siempre, que es posible, el método de autofotografía en erección propuesto por Kelami ${ }^{5}$ (Fig. 1). En los casos en que ésta no fue posible practicamos el test de inyección intracavernosa con 20 mcg de PGE-1.

\section{TABLA I}

TIPOS DE INCURVACIÓN

\begin{tabular}{|l|c|c|}
\hline Tipo de incurvación & № casos & Porcentaje \\
\hline Dorsal & 16 & $35,55 \%$ \\
\hline Dorso-lateral & 19 & $42,22 \%$ \\
\hline Lateral & 8 & $17,86 \%$ \\
\hline Ventral & 1 & $2,22 \%$ \\
\hline Ventro-lateral & 1 & $2,22 \%$ \\
\hline
\end{tabular}

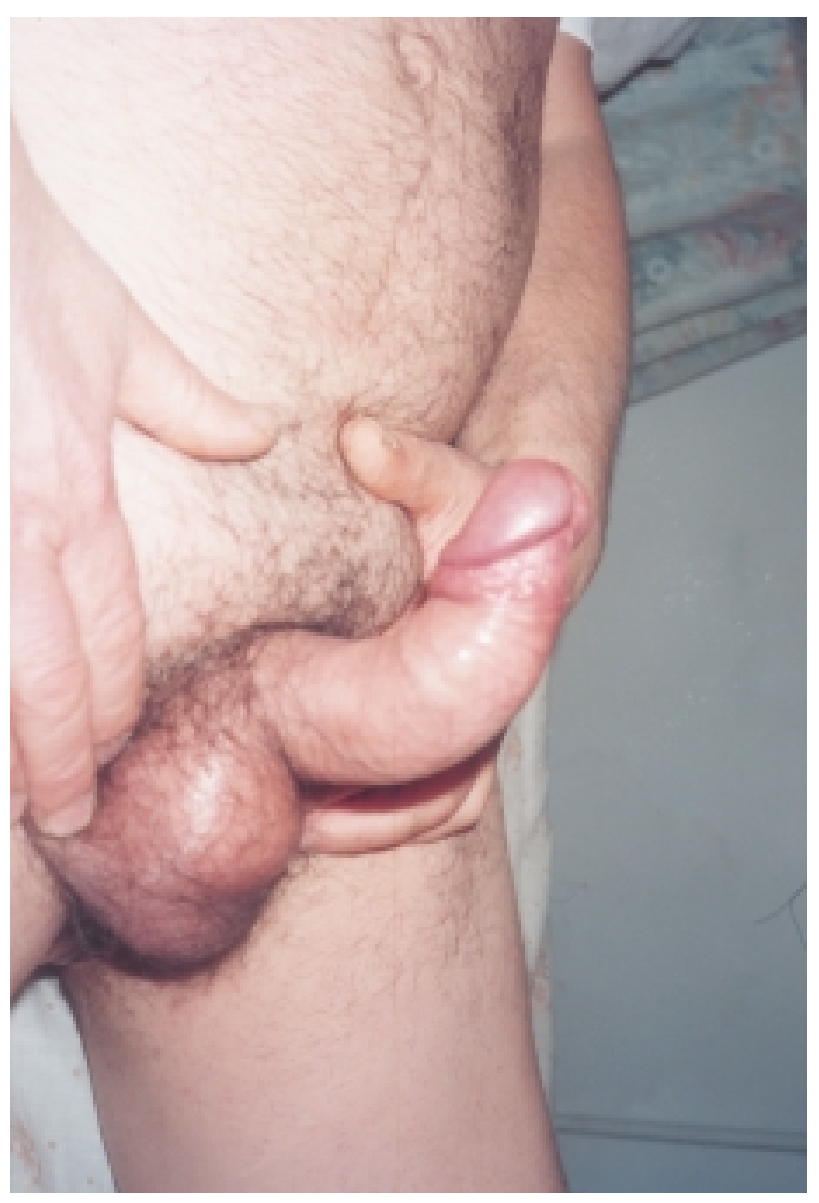

FIGURA 1. Autofotografia.

Cuando se realizaron las autofotografías (30 casos) valoramos el ángulo de incurvación mediante el sistema de 5 líneas descrito por Kelami. En la Tabla II recogemos el número de casos según el grado de incurvación. No incluimos enfermos con incurvaciones inferiores a $30^{\circ}$ ya que no las consideramos tributarias de corrección quirúrgica.

En 5 casos había DEP leve antes de la intervención $(11,36 \%)$.

En todos los pacientes se realizó la técnica de Nesbit para corregir la incurvación peneana. La secuencia quirúrgica fue la siguiente:

\section{TABLA II}

NÚMERO DE CASOS SEGÚN GRADO DE INCURVACIÓN

\begin{tabular}{|c|c|c|}
\hline Grado de incurvación & № de casos & Porcentaje \\
\hline $30^{\circ}-45^{\circ}$ & 14 & $46,67 \%$ \\
\hline $45^{\circ}-60^{\circ}$ & 16 & $53,33 \%$ \\
\hline
\end{tabular}


1. Incisión coronal en todos los pacientes, con circuncisión cuando el prepucio era redundante.

2. Disección de la piel hasta la base del pene, con exposición de la fascia de Buck.

3. Provocamos la erección artificial mediante la inyección de suero salino, a través de una aguja de Butterfly que penetra en el cuerpo cavernoso a través del glande y con un torniquete en la base del pene. De esta forma podemos valorar sobre el terreno el tipo de incurvación (Fig. 2).

4. Incisión longitudinal a nivel lateral de la fascia de Buck llegando hasta la albuginea (Fig. 3). Si la desviación es dorsal se deberá liberar hasta el límite de la uretra, pero si es ventral se liberará el paquete vasculonervioso.

5. Se realizaron una o más elipses en la cara contralateral del lugar de máxima deformidad, hasta conseguir enderezar el pene (Fig. 4). Al extirpar la elipse es importante no lesionar el tejido cavernoso subyacente.

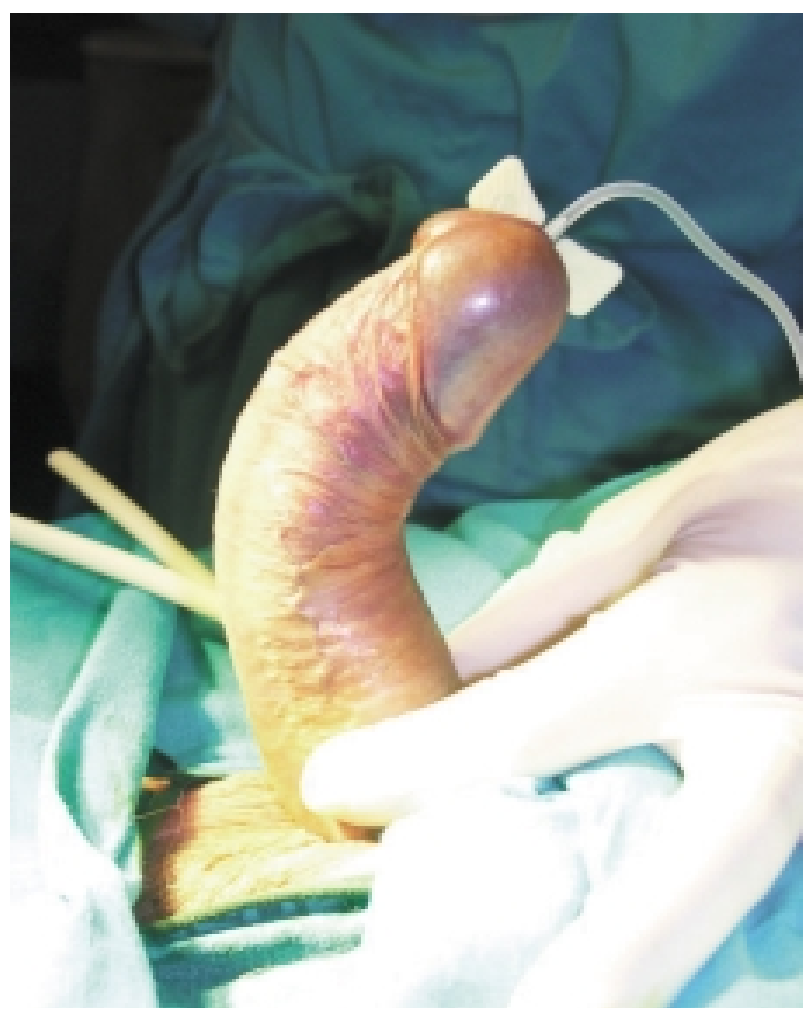

FIGURA 2. Inyección de suero salino al cuerpo cavernoso a través del glande.

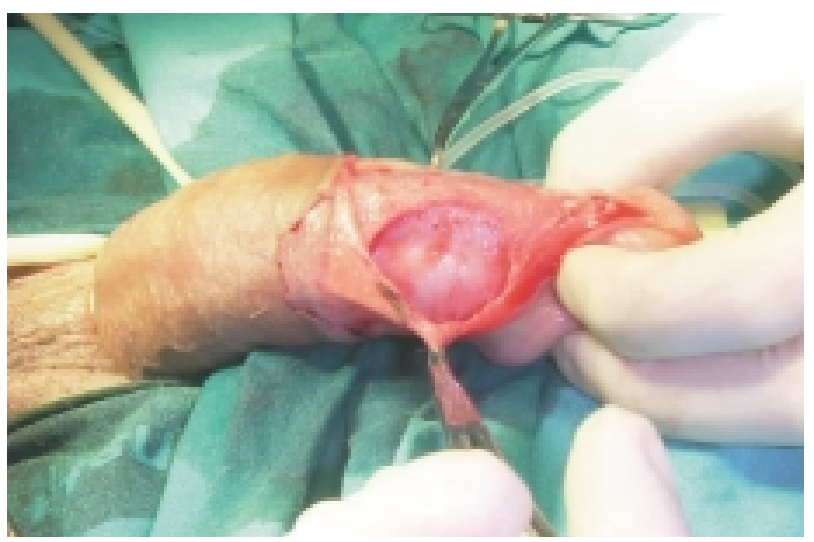

FIGURA 3. Incisión longitudinal de la fascia de Buck.

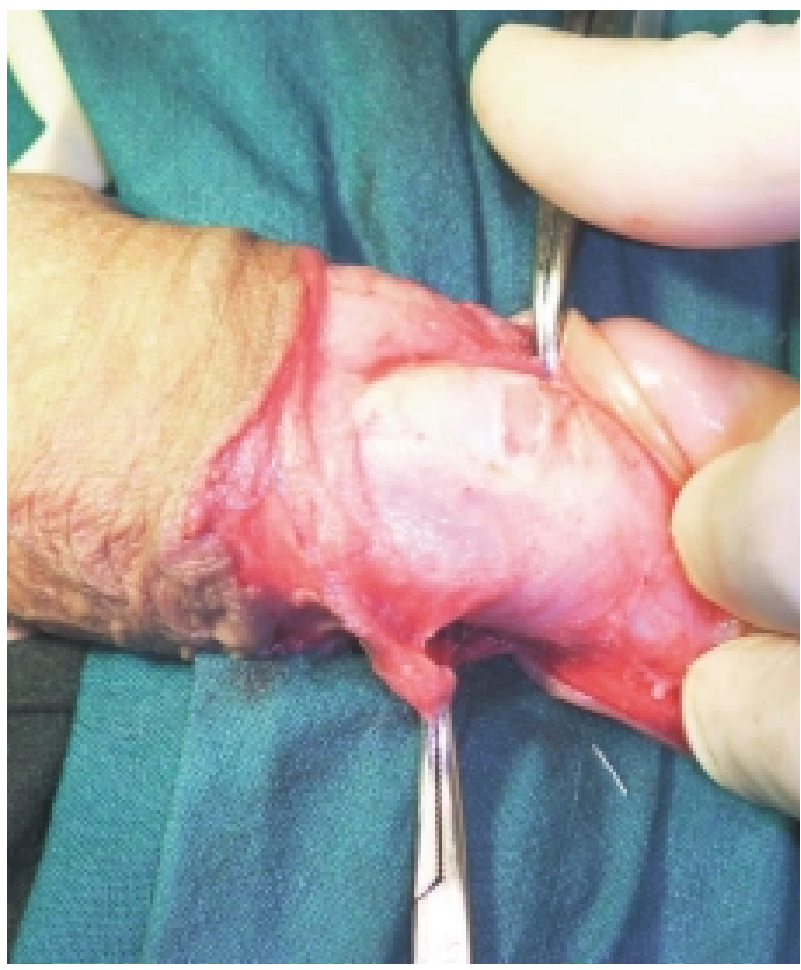

FIGURA 4. Elipse del cuerpo cavernoso.

6. Para realizar la sutura utilizamos material reabsorbible (ác. poliglicólico 3/0) y puntos simples.

7. Nueva inducción de la erección artificial para confirmar que el pene ha sido enderezado correctamente.

8. Cierre de la fascia de Buck con ác. poliglicólico 4/0. Sutura de la piel con ác. poliglicólico $4 / 0$ y vendaje compresivo del pene que dejamos durante 24 horas (Fig. 5). 


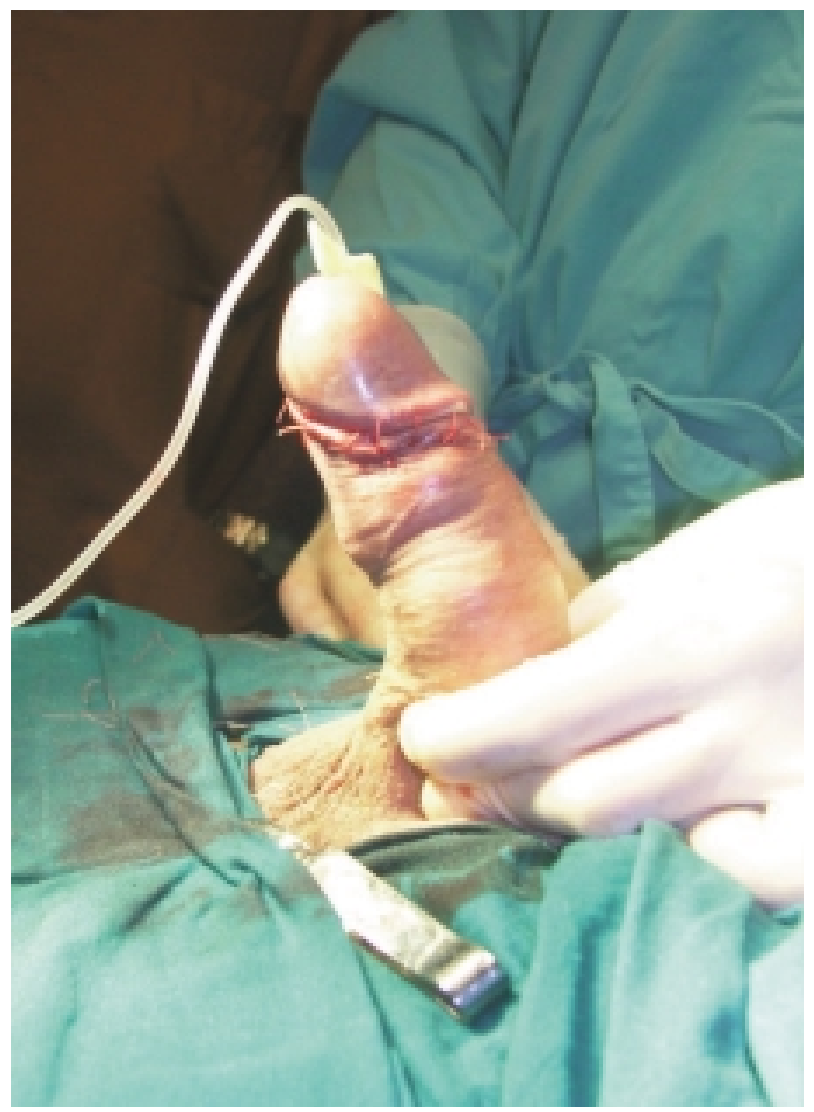

FIGURA 5. Resultado final con la incurvación corregida.

En todos los casos se realizó profilaxis antibiótica en dosis única ( $1 \mathrm{~g}$ de cefazolina), durante la inducción anestésica.

El seguimiento medio después de la cirugía fue de 63,03 meses (Rango 10-127 meses).

\section{RESULTADOS}

Se consiguió una buena corrección de la incurvación en 40 casos $(88,88 \%)$, en los otros 5 casos $(11,11 \%)$ la corrección sólo fue parcial.

En cuanto al grado de satisfacción que mostraron los pacientes después de la cirugía fue de 39 casos satisfechos $(86,66 \%)$ y 6 casos insatisfechos $(13,33 \%)$.

Los motivos de insatisfacción fueron los siguientes: En dos casos la recidiva temprana de la incurvación produjo la insatisfacción. Un caso fue por transtornos persistentes de la sensibilidad del glande (cabe destacar que hubo otros dos casos de transtornos de la sensibilidad, que al mejorar antes de los 6 meses no fueron motivo de insatisfacción). Finalmente en 3 casos la falta de erección después de la intervención fue la causa de la insatisfacción. Cabe destacar que los tres la tenían previamente y que por lo tanto no se la puede atribuir a la cirugía.

Hay que destacar que ningún paciente se mostró insatisfecho por acortamiento del pene. Las molestias en la zona de la sutura siempre fueron transitorias. Además en ningún paciente se pudo demostrar DEP, secundaria a la cirugía.

La estancia media hospitalaria fue de 3 días.

\section{DISCUSIÓN}

La técnica de Nesbit para la corrección de la incurvación peneana provocada por la enfermedad de Peyronie es un buen procedimiento, no obstante es importante una correcta valoración preoperatoria para no incluir pacientes que puedan llevar al fracaso de la técnica. En esta línea cabe resaltar que no hay que incluir nunca pacientes en etapa precoz, que aún presenten dolor durante la erección, y esperar como mínimo 6 meses a que éste haya desaparecido.

Es muy importante valorar adecuadamente la función eréctil en el periodo preoperatorio ${ }^{6}$, ya que como demuestra Porst ${ }^{7}$, el grado de satisfacción post-operatoria varía de un $61 \%$ en los que tenían disfunción eréctil preoperatoria, a un 89\% de los que no la tenían. En esta línea, nosotros en la actualidad no intervenimos, mediante la técnica de Nesbit, enfermos con DEP y que previamente no hayan respondido a un tratamiento no cruento.

Otro aspecto importante es el grado de incurvación peneana, que realizamos, por el método de autofotografías propuesto por Kelami en $1983^{5}$. Nosotros no incluimos pacientes con incurvaciones menores de $30^{\circ}$ ni mayores de $60^{\circ}$, ya que creemos que estos últimos serían candidatos a injertos y no plastias. Estamos por tanto de acuerdo con Levine en cuanto a los criterios de inclusión para las plastias que serían: pacientes potentes, con pene de longitud normal e incurvación definida alrededor de $50^{\circ}$. No cumpliendo estos criterios, sobre todo cuando la incurvación es muy acentuada, se deberían realizar múltiples elipses que producirian acortamiento significativo del pene. Para Pryor ${ }^{9}$ en una serie de 359 pacientes, el acortamiento superior a 2 centímetros se dio en 17 casos (4,7\%). No obstante, éste 
siempre es menos significativo cuando en casos con incurvaciones elevadas se realiza la incisión de la placa con injerto Montorsi ${ }^{10}$.

La técnica de Nesbit, es probablemente la más utilizada, sobre todo si incluimos sus múltiples variantes como la de Lemberger ${ }^{11}$, Yachia ${ }^{4}$, Sassine $^{12}$ y Belgrano ${ }^{13}$ en que se realizan incisiones verticales de la túnica albugínea y cierres horizontales.

Los buenos resultados son importantes, Pryor presenta un $82 \%$, pero si su experiencia la divide en dos periodos evolutivos, en el segundo alcanza el $90 \%{ }^{2}$. En esta línea estarían nuestros resultados, 88,8\% de corrección de la curvatura. En cuanto a la recidiva de la incurvación, ésta estaría entre el $0 \%$ de Prost $^{14}$ y el $21 \%$ de Licht ${ }^{15}$, pasando por el $11 \%$ de Ralph y el $4 \%$ de Poulsen ${ }^{16}$, nosotros hemos tenido dos casos en 45 enfermos, $4,44 \%$.

En cuanto al material utilizado para la sutura, en nuestra serie ha sido siempre readsorbible (ác. poliglicólico) y aunque autores como Jiméne $z^{17}$ indican que el uso del material irreabsorbible proporciona un índice de recidiva inferior, nosotros creemos que puede ocasionar más inconvenientes que ventajas, ya que estos puntos de sutura a pesar de anudarlos de forma invaginante pueden molestar durante el coito. Intentando una menor agresividad quirúrgica, aparecieron las técnicas de plicatura, Essed $1985^{18}$ y Ebbehoj $1985^{19}$, no obstante estas técnicas presentan el problema de depender de la tensión que genera el material de sutura, lo que explica los resultados desfavorables que presentan algunos autores como Poulsen ${ }^{16} 44 \%$ y Nooter $^{20} 24 \%$. Es evidente que con esta técnica el uso de material reabsorbible produciría peores resultados.

Finalmente, expondremos que los estudios comparativos entre plastia/plicatura se decantan a favor de la plastia, en especial utilizando la técnica de Nesbit, sobre todo en las series más recientes Schulthesiss $2000^{21}$. La recidiva utilizando las plicaturas puede llegar en algunas series hasta el 50\% de Brake $^{22}$, mientras que con la plastia de Nesbit no va más allá del $21 \%$ de Licht $^{15}$. La incidencia de DEP como consecuencia de la intervención suele ser muy baja. Belgrano ${ }^{13}$ tiene un $0 \%$, Licht $^{15} 4 \%$, no obstante Poulsen ${ }^{16}$ encuentra un porcentaje elevado del $23 \%$, pero posiblemente es debido a que muchos casos ya presentaban DEP antes de la intervención.

En la enfermedad de Peyronie las incurvaciones suelen ser dorsales o dorso-laterales, lo que hace que los transtornos de sensibilidad del glande debido a la liberación del paquete vasculonervioso sean muy escasos. En nuestra serie 1 de 46. No obstante sería conveniente utilizar gafas de aumento como preconiza Rodríguez Vela ${ }^{23}$ para facilitar la disección de dicho haz neuro-vascular.

\section{CONCLUSIONES}

La técnica de Nesbit para la corrección quirúrgica de la incurvación peneana secundaria a la enfermedad de Peyronie, cuando se aplica en pacientes bien seleccionados: potencia conservada, pene suficientemente largo e incurvación no superior a $60^{\circ}$, ha demostrado ser una técnica de fácil aplicación, de estancia media corta y con resultados tanto estéticos $(88,8 \%)$ como funcionales (grado de satisfacción) $(86,6 \%)$ altos.

\section{REFERENCIAS}

1. NESBIT RH.: Congenital curvature of the phallus: report of three cases with description of corrective operation. J Urol 1965; 93: 230-235.

2. PRYOR JP, FITZPATRICK JM.: A new approach to the correction of the penile deformity in Peyronie's disease. J Urol 1979; 122: 622-626.

3. KELAMI A.: Peyronie disease and surgical treatment. A new concept. Urology 1980; 15: 559-561.

4. YACHIA D.: Modified corporoplasty for the treatment of penile curvature. $J$ Urol 1990; 143: 80-82.

5. KELAMI A.: Autoradiografye in evaluation of functional penile disordes. Urology 1983; 21: 628-631.

6. CHANTADA ABAL V, RODRÍGUEZ VELA J, MARTÍNEZ PIÑEIRO L y cols.: Conferencia consenso sobre la enfermedad de Peyronie. Reunión Nacional del Grupo de Andrología. Asociación Española de Urología. Actas Urol Esp 1999; 23: 183-221.

7. PORST H.: Congenital and acquired penile derivations and penile fractures. In. Porst $\mathrm{H}$ (Ed.) Penile disorders. Springer. Germany 1997: 37-53.

8. LEVINE LA, LENTING EL.: A surgical algorithm for the treatment of Peyronie's disease. J Urol 1997; 21: 49-52.

9. PRYORT JP.: The management of Peyronie's disease. In: Porst $\mathrm{H}$ (Ed.) Penile disorders. Springer. Germany 1997: 75-86.

10. MONTORSI F, SALONIA A, MAGA T et al.: Evidence based assessment of long-term results of plaque incision and vein grafinfor Peyronie's disease. $J$ Urol 2000; 163: 1704-1708. 
11. LEMBERGER RJ, BISHOP MC, BATES CP.: Nesbit's operation for Peyronie's disease. $\mathrm{Br} J$ Urol 1984; 56: 721-723.

12. SASSINE AM, WESPES E, SCHULMAN CC.: Modified corporoplasty for penile curvature: 10 years experience. Urology 1994; 44: 419-421.

13. BELGRANO E, LIGUORI G, TROMBETTA C, SIRACUSANO S.: Correction of complex penile deformities by modified Nesbit procedure asymetric tunica albuginea excision. Eur Urol 2000; 38: 172-176.

14. PORST H.: Corporoplasty in congenital and acquired penile derivations: technique and outcome in 62 patients. Urology (A) 1989; 28: 168-171.

15. LICHT MR, LEWIS RW.: Modified Nesbit procedure for the treatment of Peyrinie's disease: a comparative outcome analysis. J Urol 1997; 158: 460-463.

16. POULSEN J, KIRKEBY HJ.: Treatment of penile curvature-a retrospective study of 175 patients operated upon with plication of the tunica albuginea or with the Nesbit procedure. $B r J$ Urol 1995; 75: 370-374.

17. JIMÉNEZ CRUZ JF, MORENO PARDO B.: Enfermedad de la Peyronie, priapismo e incurvación de pene. Ponencia Oficial al LII Congreso Nacional de Urología. Palma de Mallorca 1987.

18. ESSED E, SCHROEDER FH.: New surgical treatment for Peyronie disease. Urology 1985; 25: 582587.
19. EBBEHOJ J, METZ P.: New operattion for "Krummerik" (penile curvature). Urology 1985; 26: 76-78.

20. NOOTER RI, BOSCH JLRH, SCHODER FH.: Peyrinie's disease and penile curvature: long-term results of operative treatment with the plication procedure. Br J Urol 1994; 74: 497-500.

21. SCHULTHESISS D, MESCHI MR, HAGEMANN $J$ et al.: Congenital and acquired penile deviation treated with the essed plication method. Eur Urol 2000; 38: 167-171.

22. BRAKE M, KELLER H, LAMADE F et al.: Surgical correction of penile derivation. Nesbit vs Schroeder-Essed method. Urologe A 1999; 38: 264269.

23. RODRÍGUEZ VELA L, BENEJAM GUAL J, BORQUE FERNANDO A et al.: Tunic albuginea plication. Our experience. Libro de abstracts del "2 $2^{\text {nd }}$ Meeting of the European Society for impotence Research". Madrid (Spain), 1-4 october 1997.

Dr. J. Rodríguez Tolrá

C/ San Bruno, 31-33, $3^{\text {o }} 2^{\text {a }}$

08911 Badalona (Barcelona)

(Trabajo recibido el 11 junio de 2003) 